\title{
La morfología dental en contextos clínicos, antropológicos y forenses
}

\author{
Dental morphology in clinical, anthropological and forensic contexts
}

María A. Guiglioni ${ }^{1, a, b, f}$, Gabriela G. Bessone $e^{1, a, c, g}$, Rolando P. Juárez ${ }^{1, a, d, e, h}$

\section{RESUMEN}

El propósito de este artículo es hacer una revisión de la literatura sobre los rasgos morfológicos dentales dentro de los contextos odontológico, antropológico y forense. Estos son empleados en la estimación de las relaciones biológicas entre poblaciones y relevantes en los problemas legales. Su naturaleza puede predisponer al desarrollo de procesos patológicos, de ahí la importancia de su conocimiento para el odontólogo general.

PALABRAS CLAVE: Dentición, anatomía, odontología general, antropología física, odontología forense. (DeCS, BIREME)

\section{SUMMARY}

The purpose of this article is to review the literature on dental morphological traits within the dentistry, anthropological and forensic contexts. They are employed in the estimation of biological relations between populations and they are relevant to legal problems. Its nature may predispose the development of pathological processes, hence the importance of knowledge for the general dentist.

KEYWORDS: Dentition, anatomy, dental general practice, physical anthropology, forensic dentistry. (MeSH, $N L M)$

\footnotetext{
${ }^{1}$ Facultad de Odontología, Universidad Nacional del Nordeste. República Argentina.

a Odontólogo (a).

${ }^{\mathrm{b}}$ Especialista en Docencia Universitaria

- Especialista en Metodología de la Investigación en Ciencias de la Salud.

¿ Especialista en Salud Pública.

e Doctor en Odontología.

† Profesora Titular de la Cátedra de Introducción a la Odontología.

g Profesora Adjunta de la Cátedra de Introducción a la Odontología.

n Profesor Titular de la Cátedra de Fisiología Humana.
} 


\section{INTRODUCCIÓN}

Investigadores de varias áreas del conocimiento humano, vienen estudiando los dientes por la gran información que aportan al campo específico de las disciplinas científicas relacionadas con la morfología dental $(1,2)$.

La base metodológica principal en los estudios dentarios sobre morfología dentaria es el análisis comparado de los caracteres dentarios, en sus distintos niveles: individual, intragrupal e intergrupal (3).

Los estudios de rasgos no-métricos (odontoscopía o morfología dental), consisten en observar, registrar, analizar y comprender el comportamiento de la expresión (frecuencia y variabilidad) de la morfología coronal y radicular de los dientes humanos $(4,5)$. Son empleados en la estimación de relaciones biológicas entre poblaciones, para esclarecer los procesos históricos, culturales y biológicos evolutivos (6).

El estudio de los rasgos métricos (odontometría) consiste en la obtención de las medidas coronales y radiculares de los dientes. Estas dimensiones son útiles para la predicción de espacio durante los tratamientos ortodóncicos, son empleadas en estudios antropológicos evolutivos y comparativos y son útiles para determinar el sexo de un individuo en los procesos de identificación forenses (7).

La morfología dental y la odontometría deben ser estudiadas desde un punto de vista interdisciplinario (biología, antropología, odontología, paleopatología, arqueología, ciencias forenses) toda vez que los dientes contribuyen en el esclarecimiento del proceso evolutivo del hombre y la distribución de la conformación de los complejos dentales poblacionales (5).

\section{REVISIÓN DE LITERATURA}

En la presente revisión de literatura, dentro de los conceptos de la morfología dental, se describen los rasgos morfológicos dentales, desde sus aspectos clínicos, antropológicos y forenses.

\section{Rasgos morfológicos dentales}

La morfología de los dientes consiste en una serie de características denominadas rasgos morfológicos dentales (RMD) coronales y radiculares, con fuerte control genético que permiten el establecimiento de relaciones directas entre igualdad estructural y filiación poblacional durante la odontogénesis (3).
La estructura dental, no cambia por acción propia ni se remodela como sucede con el hueso, salvo los cambios que el diente sufre por el desgaste mecánico o atrición y acumulación de dentina reparadora (2).

El esmalte es el tejido más duro del cuerpo humano, y tiene la capacidad de soportar altas temperaturas; y además, presenta una muy alta resistencia tafonómica (paso del tiempo, medio ambiente, $\mathrm{pH}$, salinidad, humedad, ataque de elementos traza e isótopos estables) (8).

Es probable que, los grupos que posean una morfología dentaria similar estén relacionados biológicamente entre sí, pues se sugiere que la semejanza fenotípica se aproxima a la semejanza genética (9).

Así, los dientes se constituyen en indicadores precisos para el estudio de poblaciones humanas, pues se los considera marcadores y unidades de comparación de origen genético, lo cual permite clasificar a los grupos y a los individuos que los integran de acuerdo con las categorías taxonómica, filogenética y evolutiva, pero especialmente a través de la frecuencia, el dimorfismo sexual, la bilateralidad y la asociación de las características morfológicas dentales (8).

Pueden ser estructuras positivas (tuberculares y radiculares) o negativas (intertuberculares y fosomorfos), coronarios o radiculares; es decir, rasgos morfológicos dentales coronarios (RMDC) y rasgos morfológicos dentarios radiculares (RMDR). Tienen el potencial de estar o no presentes en un sitio específico (frecuencia) de diferente manera (variabilidad) en uno o más miembros de un grupo poblacional(6).

En la literatura especializada, los RMDC se reconocen como caracteres, particularidades, variantes, aspectos, atributos, polimorfismos, anomalías, rasgos discretos o epigenéticos o expresiones fenotípicas (10). Estudios sobre la heredabilidad de los RMDC, en diferentes grupos humanos, permiten que no se sigan catalogando a los RMDC como anomalías de forma, sobre todo en el contexto odontológico universitario, ya que son características morfológicas y fenotípicas de la dentición, evidenciadas en su alta frecuencia y variabilidad de expresión $(5,11,12)$.

La función de masticación, no es la responsable de la difusión de los RMD en una determinada población. Sin embargo, algunos autores refieren que la morfología del diente (patrón de depresiones y elevaciones) sobre todo en los dientes posteriores (premolares y molares), así como el espesor del esmalte, están en relación con 
la dieta, ya que las propiedades físicas de la comida demandan una determinada relación interdentaria y definen, a través de la selección por adaptación de la función, ciertas características de patrones oclusales $(13,14)$

En la dentición humana se han reconocido más de 100 rasgos morfológicos dentales, pero en la mayoría de las investigaciones internacionales se emplean no más de diecisiete rasgos, principalmente los que se encuentran ubicados en la corona de los incisivos y en los molares de ambas denticiones $(5,8,15)$. Algunos de estos rasgos son: winging, incisivos centrales y laterales en pala, doble pala, cúspide de Carabelli, patrón cuspídeo, número de cúspides, cresta distal del trigónido, pliegue acodado, protostílido, cúspide 6 y cúspide 7 (12).

El análisis de series prehistóricas realizadas en Argentina, en pueblos originarios del noroeste, suroeste y nordeste, mostraron rasgos morfológicos dentales similares, con una relación inclusiva de esta muestra dentro del patrón dental mongoloide sinodonte propuesto para las poblaciones amerindias de América (16-18). Si comparamos las colecciones estudiadas observamos un agrupamiento muy homogéneo a las frecuencias de los incisivos en pala, con frecuencias altas de este rasgo en todas las series estudiadas (3).

Los caracteres dentales presentan una serie de ventajas frente a los rasgos observados en los restos óseos, estas son: un fuerte componente genético y una elevada heredabilidad, una sencilla observación con un entrenamiento previo (incluso habiendo desgaste dental) y finalmente una mejor conservación y preservación, ya que las piezas dentales perduran más que los huesos (19).

Además de su gran valor antropológico y forense, algunos de estos rasgos resultan de gran importancia clínica en el contexto odontológico, ya que por su naturaleza pueden predisponer o favorecer el desarrollo de algún proceso patológico, en cuyo caso el plan de tratamiento debe partir de un correcto diagnóstico basado en el conocimiento del comportamiento del rasgo como factor etiológico (2).

De acuerdo a la morfología de las superficies de los dientes, existen sitios específicos que favorecen la retención de placa dental y por lo tanto, se constituyen en nichos ecológicos que propician la proclividad de la caries, favorecidos de igual forma por la capacidad de las superficies sólidas, como el esmalte, de coadyuvar en la formación de la biopelícula, la cual incrementará su retención con base a la rugosidad, el área y las características topográficas de dicha superficie dental (20).

Algunos rasgos morfológicos dentales coronales y radiculares, como es el caso del surco interrumpido, las extensiones de esmalte y las perlas de esmalte, se ven involucrados en la etiología de la enfermedad periodontal, por lo tanto el estudio del comportamiento de estos rasgos desde el punto de vista de la antropología dental podría, eventualmente, guiar los procedimientos clínicos a partir de la comprensión de la expresión de los mismos, considerando éstos como una variación morfológica normal de los grupos humanos (21).

\section{DISCUSIÓN}

Comprender el mundo natural no es posible sin conocer ampliamente la morfología de los seres vivos y sus sistemas, pues por su gran utilidad ilustrativa es aplicable en muchas áreas biológicas como la odontología.

Evidenciar el comportamiento de la expresión de la morfología dentaria presenta significativos alcances clínicos, étnicos y forenses. En la clínica odontológica la morfología funcional cumple un papel determinante en las relaciones oclusales durante las funciones del sistema estomatognático. En los contextos antropológico y forense, debido a que el análisis de la morfología de los dientes contribuye con el establecimiento de la cuarteta básica de identificación (osterografía u odontografía) específicamente en la estimación de la edad, sexo y patrón étnico (22-24).

Actualmente, se acepta que la morfogénesis de la corona dental está controlada en su mayor parte por factores genéticos, más que por factores ambientales, situación que revaloriza la dentición para estudios y análisis poblacionales (13).

Caracterizando la poblacion estudiada, se determina afinidades con otras poblaciones, pudiendo develar patrones de filiación biológica en poblaciones antiguas, procesos microevolutivos, hábitos alimenticios y tecnologías en el procesamiento de alimentos (25).

Por otro lado, las propiedades biomecánicas de las piezas dentarias traen consigo una mayor preservacion respecto a otros tejidos del cuerpo, lo que las hace de un valor importante como elemento descriptivo y analítico en estudios antropológicos (18). 


\section{CONCLUSIONES}

Todos los aspectos señalados nos brindan elementos suficientes para ayudar a resolver algunas problemáticas históricas de nuestros pueblos originarios, en el marco de enfoques bioculturales. La información obtenida desde la antropología dental también es útil para la odontología clínica y la forense.

Sin embargo, en Suramérica han sido exiguos los estudios que han empleado información dental para resolver problemáticas antropológicas, clínicas y/o forenses. No obstante al ser una temática interesante y casi inexplorada, debe ser abordada por futuras investigaciones.

\section{Correspondencia:}

Rolando Juárez.

Avenida Libertad 5450, Campus Universitario.

(3400) Corrientes, Argentina.

Correo electrónico: ropablojuarez@gmail.com ropablojuarez@odn.unne.edu.ar

\section{REFERENCIAS BIBLIOGRÁFICAS}

1. Alt KW, Teschler-Nicola M, Rösing FW. Dental anthropology. Fundamentals, limits and prospects. Berlin: Springer Verlag; 1998.

2. Moreno S, Moreno F. Importancia clínica de la antropología dental. Rev Estomat. 2007;15 Supl 2:4253.

3. Bollini GA, Rodríguez-Flórez CD, Colantonio SE, Méndez MG. Morfología dental de una serie prehistórica de araucanos provenientes de la Patagonia argentina y su relación biológica con otras poblaciones prehistóricas argentinas y del mundo. Int J Morphol. 2006; 24(4):705-12.

4. Rodríguez CD. La antropología dental y su importancia en el estudio de los grupos humanos. Rev Fac Odont Univ Ant. 2005; 16(1-2):52-9.

5. Girón G, Gómez P, Morales L, León M, Moreno F. Rasgos morfológicos y métricos dentales coronales de premolares superiores e inferiores en escolares de tres instituciones educativas de Cali, Colombia. Int J Morphol. 2009; 27(3):913-25.

6. Rodríguez JV. Dientes y diversidad humana: avances de la antropología dental. Santa Fe de Bogotá,Colombia: Universidad Nacional de Colombia; 2003.

7. Rodríguez JV. La antropología forense en la identificación humana. Santa Fe de Bogotá, Colombia: Universidad Nacional de Colombia; 2004.

8. Aguirre L, Castillo D, Solarte D, Moyano M, Moreno F. Morfología dental en dentición mixta: correlación de tres rasgos morfológicos en dientes temporales y permanentes de escolares de Cali, Colombia. Revista Estomatología. 2007; 15(2):10-8.

9. Artaria M. The dental traits of Indonesian Javanese. Dent Anthropol J. 2010; 23(3):74-8.

10. Moreno F, Moreno SM, Díaz CA, Bustos EA, Rodríguez JV. Prevalencia y variabilidad de ocho rasgos morfológicos dentales en jóvenes de tres colegios de Cali, 2002. Colomb Med. 2004; 35 Supl 1:16-23.

11. Manabe Y, Oyamada J, Kitagawa Y, Rokutanda A, Kato K, Matsushita T. Dental morphology of the Dawenkou Neolithic population in North China: implications for the origin and distribution of Sinodonty. J Hum Evol. 2003; 45(5):369-80.

12. Aragón N, Bastidas C, Bedón LK, et al. Rasgos morfológicos dentales coronales en dentición temporal y permanente: distancia biológica entre tres grupos indígenas del Amazonas Colombiano. Revista Odontológica Mexicana. 2008; 12(1):13-28.

13. Kono RT. Molar enamel thickness and distribution patterns in extant great apes and humans: new insights based on a 3-dimensional whole crown perspective. Anthropol Sci. 2004;112:121-46.

14. Reyes G, Bonomie J, Guevara E, et al. El sistema dental y su importancia en el estudio de la evolución humana: revisión bibliográfica. Boletín Antropológico. 2010;1(78):16-43.

15. Moreno SM, Moreno F. Eight non-metric dental traits in alive racially mixed population from Cali, Colombia. Int J Dental Anthropol. 2005; 6:14-25.

16. Scott RG, Turner CG. The anthropology of modern human teeth. Londres: Cambridge University Press; 2000.

17. Bollini GA, Rodríguez-Flórez CD, Colantonio SA. Morfología dental en una muestra de cráneos humanos de Pampa Grande, Argentina. Int J Morphol. 2010; 28(3):685-96.

18. Bollini GA, Rodríguez-Flórez CD, Colantonio S. Morfología dental en cráneos Toba de Argentina. Revista AntropoGenetika. 2010; 21:27-38.

19. Ruiz J, Muncunill J, Fontanals-Coll M, Subirà ME. Morfología dental en poblaciones mesolíticas y neolíticas del Mediterráneo. Una aproximación a las relaciones filogenéticas entre poblaciones mesolíticas y neolíticas del levante peninsular a partir de la morfología dental. Revista del Museu de Gavà. 2012; 5:79-85.

20. Moreno S, Villavicencio J, Ortiz M, Jaramillo A, Moreno F. Restauraciones preventivas en resina como estrategia para control de la morfología dental. Acta Odontol Venez. 2007; 45(4):580-8.

21. Soto J, Moreno S, Moreno F. Antropología dental y periodoncia: relación entre los rasgos morfológicos dentales y la enfermedad periodontal. Acta Odontol Venez. 2010; 48(3):1-13.

22. Figún ME, Garino RR. Anatomía odontológica: funcional y aplicada. 2da. edición. Buenos Aires: El Ateneo; 2002.

23. Moreno S, Moreno F. Antropología dental: una herramienta valiosa para fines forenses. Revista 
estomatol. 2002; 10(2):29-42.

24. Edgar HJ. Prediction of race using characteristics of dental morphology. J Forensic Sci. 2005; 50(2):1-5.

25. Rodríguez-Flórez CD. La antropología dental y su importancia en el estudio de los grupos humanos prehispánicos. RAE (Universidad de Jaén, España). 2004; 4:1-7.

Recibido: 28/05/2014

Aceptado: 04/07/2014 